\title{
Influence of increased arterial stiffness on myocardial work efficiency in patients with stable coronary artery disease
}

\author{
Mateusz Dziarmaga1, Andrzej Minczykowski ${ }^{1}$ Marcin Zwanzig1, Tomasz Krauze ${ }^{1}$, Agnieszka Rutkowska1, \\ Jan Morawski ${ }^{1}$, Marek Baliński ${ }^{1}$ Jarosław Piskorski², Przemysław Guzik ${ }^{1}$ Andrzej Wykrętowicz \\ 1 Department of Cardiology-Intensive Therapy, Poznań University School of Medicine, Poznań, Poland \\ 2 Faculty of Physics and Astronomy, University of Zielona Góra, Zielona Góra, Poland
}

Correspondence to: Andrzej Wykrętowicz, MD, PhD, Department of Cardiology-Intensive Therapy, University School of Medicine, ul. Przybyszewskiego 49, 60-355 Poznań, Poland phone: +48618691391 email: awykreto@ptkardio.pl Received: November 8, 2020. Revision accepted: January 18, 2021. Published online: January 27, 2021. Kardiol Pol. 2021; 79 (2): 196-198 doi:10.33963/KP.15784

Copyright by the Author(s), 2021
Introduction The analysis of the left ventricular (LV) pressure-strain loops quantifies different features of myocardial work and helps to understand energy expenditure related to blood ejection to the aorta. ${ }^{1-6}$ The global constructive work (GCW) represents energy spent by viable cardiomyocytes on contracting and shortening of LV segments during mechanical systole and their lengthening during isovolumetric relaxation. ${ }^{2-5}$ Contrary, if some segments elongate during LV systole and shorten during isovolumetric relaxation, such energetic cost is considered as the global wasted work (GWW) as it does not participate in the ejection. The ratio of the GCW to the global work index (GWI) (the sum of GCW and GWW) performed by the LV is termed the global work efficiency (GWE). Patients with LV systolic dysfunction have lower GWE. ${ }^{4,7}$

Myocardial work depends on the LV contractility and geometry change, and is also modulated by factors affecting the LV function..$^{1-4,7}$ Arterial load (AL) is an example: it represents net opposing forces that LV needs to overcome during ejection. ${ }^{8-10}$ Arterial stiffness, the most relevant contributor to the $\mathrm{AL}$, increases in patients with cardiovascular risk factors and diseases. ${ }^{8-15}$ However, the dependence of the myocardial work on arterial stiffness is uncertain. For this reason, we assessed the relationship between indices of myocardial work and pulse wave velocity (PWV), an indirect measure of arterial stiffness, in patients with stable coronary artery disease (CAD).
Patients and methods A total of 89 consecutive adult patients (61 men) with stable CAD referred for coronary angiography were recruited to the study. The local Ethics Committee approved the study protocol, and all patients gave their informed, voluntary, and written consent for participation.

In addition to regular clinical assessment, all patients underwent: 1) Standard transthoracic echocardiography (Vivid E95; GE Healthcare, Horten, Norway) for LV wall thickness, systolic and diastolic volumes, and function measurement. Additionally, speckle tracking analysis was performed to quantify GWC, GWW, and GWE with the use of the Automated Function Imaging (AFI) software (GE Healthcare, Horten, Norway). ${ }^{1,2,4}$ 2) Simultaneous Doppler echocardiographic (Sonosite 5500, Philips, The Netherlands) and applanation tonometry (NIHem, Cardiovascular Engineering Inc., Norwood, Massachusetts, United States) measured the pulse wave propagation time between the opening of the aortic valve and femoral arteries and PWV.

Statistical analysis Continuous data distribution (the Shapiro-Wilk test) was normal; thus, results are presented as mean (SD). The rates of binomial data are presented as percentages. The multiple linear regressions for the relation between myocardial work indices (dependent variables) and PWV (a single independent variable) were adjusted for the following covariates: patients' age, sex, LV ejection fraction (LVEF), the ratio between early mitral inflow velocity 
TABLE 1 Multiple linear regression models adjusted for patient's age, sex, left ventricular ejection fraction, the ratio between early mitral inflow velocity and mitral annular early diastolic velocity, and resting heart rate, for the dependence of the global work efficiency or global constructive work, or global wasted work or global work index on the pulse wave velocity in patients with stable coronary artery disease

\begin{tabular}{|c|c|c|c|c|c|c|c|c|c|}
\hline & & \multicolumn{2}{|c|}{ GWE } & \multicolumn{2}{|c|}{ GCW } & \multicolumn{2}{|c|}{ GWW } & \multicolumn{2}{|l|}{ GWI } \\
\hline & & Slope (SE) & $P$ value & Slope (SE) & $P$ value & Slope (SE) & $P$ value & Slope (SE) & $P$ value \\
\hline \multirow{6}{*}{$\begin{array}{l}\text { Adjusting } \\
\text { factors }\end{array}$} & Age, y & $0.06(0.05)$ & 0.23 & $9.18(5.37)$ & 0.091 & $1.28(1.11)$ & 0.25 & $9.13(4.23)$ & 0.03 \\
\hline & Sex (0/1 women/men) & $-0.23(0.99)$ & 0.82 & $-277.44(106)$ & 0.01 & $-43.26(21.98)$ & 0.052 & $-193.12(83.54)$ & 0.02 \\
\hline & Heart rate, bpm & $-0.15(0.04)$ & $<0.001$ & $-15.07(4.54)$ & 0.001 & $1.92(0.94)$ & 0.045 & $-16.04(3.58)$ & $<0.001$ \\
\hline & LVEF, \% & $0.13(0.04)$ & 0.001 & $12.31(4.13)$ & 0.004 & $-1.07(0.86)$ & 0.21 & $12.42(3.26)$ & $<0.001$ \\
\hline & $E / e^{\prime}$ & $-0.66(0.12)$ & $<0.001$ & $-34.02(13.16)$ & 0.01 & $2.16(2.72)$ & 0.43 & $-29.86(10.37)$ & 0.005 \\
\hline & $\mathrm{PWV}, \mathrm{m} / \mathrm{s}$ & $-0.30(0.14)$ & 0.04 & $-29.34(15.22)$ & 0.058 & $0.93(3.16)$ & 0.77 & $-30.37(12)$ & 0.013 \\
\hline \multicolumn{2}{|c|}{$R^{2}$ for the model } & \multicolumn{2}{|c|}{0.49} & \multicolumn{2}{|l|}{0.42} & \multicolumn{2}{|l|}{0.12} & \multicolumn{2}{|l|}{0.51} \\
\hline
\end{tabular}

Abbreviations: E/e', the ratio between early mitral inflow velocity and mitral annular early diastolic velocity; GCW, global myocardial constructive work; GWE, global myocardial work efficiency; GWI, global myocardial work index; GWW, global myocardial wasted work; LVEF, left ventricular ejection fraction; PWV, pulse wave velocity; SE, standard error

and mitral annular early diastolic velocity (E/e'), and resting heart rate. A $P$ value of less than 0.05 was considered statistically significant. All analyses were performed with SPSS Statistics for Windows, version 23.0 (Released 2015, IBM Corp., Armonk, New York, United States).

Results and discussion The mean (SD) age of patients was 63.3 (9.3) years. They were overweight, with preserved LVEF, and had a mean (SD) borderline global longitudinal peak systolic strain of $-16.5 \%$ (4\%). The majority of patients had hypertension. All patients were pharmacologically treated and achieved target values of less than $140 \mathrm{~mm} \mathrm{Hg}$ for systolic and less than $90 \mathrm{~mm} \mathrm{Hg}$ for diastolic blood pressure. Approximately one-third of the study patients underwent some form of myocardial revascularization (percutaneous coronary intervention or coronary artery bypass grafting), and $25 \%$ survived myocardial infarction. While the mean LVEF was within the normal range, the value of global longitudinal peak systolic strain was significantly reduced. All patients were on at least one antiplatelet drug, nearly all on a statin, a majority on a $\beta$-blocker, and either angiotensin enzyme inhibitor or angiotensin II receptor blocker. Details on all clinical characteristics of the study patients are shown in Table S1 in Supplementary material.

The linear regression models (TABLE 1) showed that GWE and GWI were significantly and negatively related to PWV in patients with stable CAD. The relation between GWC and PWV was borderline significant suggesting a trend. No association was observed for GWW and PWV.

In statistically significant models, increasing PWV was accompanied by a reduction in either GWE or GWI, both of which translate into a worse use of cardiac energy for the ejection of blood by the LV to the aorta. These effects were independent of patients' age, sex, indices of systolic (LVEF), and diastolic (E/e') LV function, and resting heart rate. Figure $S 1$ in Supplementary material presents the linear regressions for the dependence of GWE, GCW, GWW, and GWI to PWV.

Risk factors for CAD, LV impairment, and arterial stiffness vastly overlap. Advancing age, hypertension, diabetes, smoking, or obesity/overweight have substantial impact on these clinical problems. A typical example of atherosclerosis, $\mathrm{CAD}$, is a common cause of $\mathrm{LV}$ dysfunction and heart failure (HF) and is often accompanied by an increased arterial stiffness. ${ }^{4,6,11-14}$

Very recently, various studies have reported a reduction in indices of myocardial work in patients with significant CAD,${ }^{6}$ non-ST-segment elevation-acute coronary syndrome, particularly those with acute coronary occlusion. ${ }^{3}$ Chan et al ${ }^{4}$ found lower values of myocardial work indices in patients with both ischemic and nonischemic cardiomyopathy. Further, Galli et $\mathrm{al}^{7}$ observed that more reduced GCW was present in patients with severe HF who were nonresponders to cardiac resynchronization therapy.

Repeated episodes of ischemia leading to myocardial stunning or injury, gradual loss of viable myocardium, and development of scars are the potential mechanisms responsible for the reduction of myocardial work indices in patients with CAD or HF..$^{2-7}$ However, during ejection, the contracting LV must overcome the opposing effects of the arterial load.

With this observational study, we show that there is a significant association between increased arterial load and myocardial work indices in patients with CAD. Lower GWI and GWE accompany increased arterial stiffness, and this effect is independent of LVEF, E/e', resting heart rate as well as patient's age and sex.

If arterial stiffness was reduced, it might improve the efficiency of myocardial work and 
presumably lower myocardial oxygen demands. Such effects are desired in patients with CAD. Whether any intervention designed to reduce arterial stiffness would be beneficial for the global work index and its efficiency requires future studies.

The same multiple linear regression models (TABLE 1) can also be analyzed from another perspective with PWV as an adjusting factor for LVEF, E/e' and heart rate as indices of cardiac function. Such analysis shows that the relations between GWE, GCW, or GWI and LVEF or $\mathrm{E} / \mathrm{e}^{\prime}$ or heart rate were statistically significant, regardless of the effects of PWV. The values of myocardial work indices were decreasing with faster resting heart rate, increasing E/e', and declining LVEF. Of note, the global wasted work was significantly raised only with increasing resting heart rate.

In summary, we showed that an increased arterial stiffness has a deleterious effect on the GWE or GWI in CAD patients.

\section{SUPPLEMENTARY MATERIAL}

Supplementary material is available at www.mp.pl/kardiologiapolska.

\section{ARTICLE INFORMATION}

CONFLICT OF INTEREST None declared.

OPEN ACCESS This is an Open Access article distributed under the terms of the Creative Commons Attribution-NonCommercial-NoDerivatives 4.0 International License (CC BY-NC-ND 4.0), allowing third parties to download articles and share them with others, provided the original work is properly cited, not changed in any way, distributed under the same license, and used for noncommercial purposes only. For commercial use, please contact the journal office at kardiologiapolska@ptkardio.pl.

HOW TO CITE Dziarmaga M, Minczykowski A, Zwanzig M, et al. Influence of increased arterial stiffness on myocardial work efficiency in patients with stable coronary artery disease. Kardiol Pol. 2021; 79: 196-198. doi:10.33963/KP.15784

\section{REFERENCES}

1 Russell K, Eriksen M, Aaberge L, et al. A novel clinical method for quantification of regional left ventricular pressure-strain loop area: a noninvasive index of myocardial work. Eur Heart J. 2012; 33: 724-733.

2 Boe E, Skulstad H, Smiseth OA. Myocardial work by echocardiography: a novel method ready for clinical testing. Eur Heart J Cardiovasc Imaging. 2019; 20: 18-20.

3 Boe E, Russell K, Eek C, et al. Noninvasive myocardial work index identifies acute coronary occlusion in patients with non-ST-segment elevation-acute coronary syndrome. Eur Heart J Cardiovasc Imaging. 2015; 16: 1247-1255.

4 Chan J, Edwards NFA, Khandheria BK, et al. A new approach to assess myocardial work by non-invasive left ventricular pressure-strain relations in hypertension and dilated cardiomyopathy. Eur Heart J Cardiovasc Imaging. 2019; 20:31-39.

5 Morbach C, Sahiti F, Tiffe T, et al. Myocardial work - correlation patterns and reference values from the population-based STAAB cohort study. PLOS One. 2020; 15: e0239684.

6 Edwards NFA, Scalia GM, Shiino K, et al. Global Myocardial Work Is Superior to Global Longitudinal Strain to Predict Significant Coronary Artery Disease in Patients With Normal Left Ventricular Function and Wall Motion. J Am Soc Echocardiogr. 2019; 32: 947-957.

7 Galli E, Leclercq C, Hubert A, et al. Role of myocardial constructive work in the identification of responders to CRT. Eur Heart J Cardiovasc Imaging. 2018; 19: 1010-1018.

8 Ooi H, Chung W, Biolo A. Arterial stiffness and vascular load in heart failure. Congest Heart Fail. 2008; 14: 31-36.

9 Zamani P, Lilly SM, Segers P, et al. Pulsatile load components, resistive load and incident heart failure: The Multi-Ethnic Study of Atherosclerosis (MESA). J Card Fail. 2016; 22: 988-995.

10 Krauze T, Cebrowska K, Schneider A, et al. Association of muscular strength with pulsatile and steady hemodynamics in patients with acute myocardial infarction. Pol Arch Intern Med. 2020; 130: 512-519.

11 Vlachopoulos C, Aznaouridis K, Stefanadis C. Prediction of cardiovascular events and all-cause mortality with arterial stiffness: a systematic review and meta-analysis. J Am Coll Cardiol. 2010; 55: 1318-1327.
12 Townsend RR, Wilkinson IB, Schiffrin EL, et al. American Heart Association Council on Hypertension. Recommendations for Improving and Standardizing Vascular Research on Arterial Stiffness: A Scientific Statement From the American Heart Association. Hypertension. 2015; 66: 698-722.

13 Yu WC, Chuang SY, Lin YP, Chen CH. Brachial-ankle vs carotid-femoral pulse wave velocity as a determinant of cardiovascular structure and function. J Hum Hypertens. 2008; 22: 24-31.

14 Borlaug BA, Kass DA, Ventricular-vascular interaction in heart failure. Heart Fail Clin. 2008; 4: 23-36.

15 Kikuya M, Asayama K, Ohkubo T. Blood pressure variability and arterial stiffness parameters derived from ambulatory blood pressure monitoring. Kardiol Pol. 2019; 77: 509-514. 